\title{
Prolonged Duration Local Anesthesia by Combined Delivery of Capsaicin- and Tetrodotoxin-Loaded Liposomes
}

\section{Permanent link}

http://nrs.harvard.edu/urn-3:HUL.InstRepos:39712832

\section{Terms of Use}

This article was downloaded from Harvard University's DASH repository, and is made available under the terms and conditions applicable to Other Posted Material, as set forth at http:// nrs.harvard.edu/urn-3:HUL.InstRepos:dash.current.terms-of-use\#LAA

\section{Share Your Story}

The Harvard community has made this article openly available.

Please share how this access benefits you. Submit a story.

Accessibility 


\section{Table of Contents}

Title 1

Table of Contents 2

Abstract 3

Lay Summary 4

Introduction $\quad 5$

Methods 6

$\begin{array}{ll}\text { Animal care } & 6\end{array}$

Sciatic blockade technique $\quad 6$

$\begin{array}{ll}\text { Assessment of nerve blockade } & 7\end{array}$

$\begin{array}{ll}\text { Solutions of capsaicin and tetrodotoxin } & 7\end{array}$

Liposome preparation $\quad 8$

$\begin{array}{ll}\text { Liposome characterization } & 8\end{array}$

Tissue harvesting and toxicity studies $\quad 9$

$\begin{array}{ll}\text { Statistical analysis } & 11\end{array}$

Results 13

$\begin{array}{ll}\text { Liposome production and characterization } & 13\end{array}$

Sciatic nerve block by free and liposomal capsaicin 15

Sciatic nerve block by free and liposomal tetrodotoxin 17

Sensory nerve block from TTX liposomes co-injected with capsaicin liposomes 19

Functional specificity of liposomal types $\quad 19$

Effect of free drugs on nerve block from liposome-encapsulated drugs 22

$\begin{array}{ll}\text { Tissue reaction } & 24\end{array}$

$\begin{array}{ll}\text { Discussion } & 28\end{array}$

$\begin{array}{ll}\text { Conclusion } & 30\end{array}$

References $\quad 31$

Glossary of Abbreviations $\quad 37$ 


\begin{abstract}
Local anesthetics are commonly used in peripheral nerve blockade for the management of postoperative and chronic pain. However, single administrations of conventional local anesthetics often result in nerve blocks that are short compared to the duration of postoperative care, and are often associated with local toxicity or systemic side effects. Consequently, there is considerable interest in developing longer-lasting and safer local anesthetic formulations.
\end{abstract}

Capsaicin, the active component of chili peppers, can produce long, sensory-selective peripheral nerve blockade, and its co-administration with tetrodotoxin (TTX), a site 1 sodium channel blocker, can achieve a synergistic effect on duration of nerve blocks. However, in sufficiently large amounts, capsaicin can be neurotoxic, and TTX can cause systemic toxicity. We evaluated whether co-delivery of small amounts of capsaicin and TTX, by their sustained release from liposomes, could achieve prolonged local anesthesia without local or systemic toxicity.

We developed capsaicin- and TTX-loaded liposomes, and injected male SpragueDawley rats with free capsaicin, capsaicin liposomes, free TTX, TTX liposomes, and blank liposomes, singly or in combination. We used a modified hotplate test and a weight-bearing test to assess the duration of sensory and motor blocks, respectively. Finally, we examined tissues microscopically and assessed the animals' rates of contralateral blockade, to determine local and systemic toxicity, respectively.

The combination of capsaicin liposomes and TTX liposomes achieved a mean duration of sensory block of 18.2 (3.8) hours [mean (SD)], far longer than from capsaicin liposomes [0.4 (0.5) hours] $(\mathrm{P}<0.001)$ or TTX liposomes [0.4 (0.7) hours] $(\mathrm{P}<0.001)$ given separately, with or without the second drug in free solution. This combination caused minimal myotoxicity and muscle inflammation, and there were no changes in the percentage or diameter of unmyelinated axons. There was no detectable systemic toxicity. 
Our results indicate that capsaicin may be useful for its synergistic effects on other formulations even when used in very small, safe quantities. This work paves the way for the development of safer and more effective local anesthetics.

\section{Lay summary}

Conventional drugs used for local anesthesia after surgery are known to produce short analgesic effects compared to the duration of recovery from surgery. Additionally, these drugs often have toxic effects to local tissues, as well as cardiovascular and neurologic side effects. Given these limitations, there is significant interest in developing a safer and more effective method for local anesthesia, for use in recovering surgical patients and other clinical applications. Previous work by our group had revealed that the combination of capsaicin - the active ingredient in chili peppers - and tetrodotoxin - the poison released by several types of puffer fish - can be injected in combination to produce long anesthetic effects in rats. However, the duration of those effects is limited by the potential toxic effects that both capsaicin and tetrodotoxin can result in, when used in sufficiently large amounts. Therefore, we set out to explore whether capsaicin and tetrodotoxin could be combined in a system that releases both drugs very slowly, for a prolonged duration of time, to achieve long local anesthetic effects without any toxicity. We developed microsized particles containing either drug and injected them in combination into the paws of rats. We tested the rats at multiple time points after injecting them by placing their paws on a hotplate and determining how long they could withstand the heat before retracting their paw, at each time point; rats that did not retract their paws within a few seconds were considered to be locally anesthetized. Our combination of particle-enclosed drugs achieved an anesthetic effect of about 18 hours. Additionally, we examined rats' tissues using microscopy but were unable to detect any toxic effects. Our results suggest that our new formulation may represent a safe and effective method for local anesthesia. 


\section{Introduction}

Local anesthetics are commonly used in peripheral nerve blockade for management of postoperative pain. However, single administrations of conventional local anesthetics often result in nerve blocks that are short compared to the duration of postoperative care. ${ }^{1}$ Moreover, local anesthetics frequently cause local toxicity affecting muscle ${ }^{2}$ and peripheral nerves, ${ }^{3}$ in direct relationship to their concentration and duration of exposure, as well as systemic toxicity affecting the cardiovascular ${ }^{4}$ and central nervous systems. ${ }^{5}$ Given these limitations, there is significant interest in developing local anesthetic formulations that can produce long lasting and safe nerve blocks.

Capsaicin (8-methyl-N-vanillyl-6-nonenamide), the active component of chili peppers, ${ }^{6-8}$ produces sensory-selective analgesia. ${ }^{6,9,10}$ It selectively binds to the TRPV1 ion channel, which is expressed on primary afferent nociceptors and enables their depolarization and excitation, leading to nociceptive responses; after initial excitation, prolonged exposure to capsaicin appears to desensitize the TRPV1 channel, resulting in analgesia. ${ }^{9,11-13}$ At low concentrations of capsaicin, nerve blockade is relatively brief. However, the duration of capsaicin-induced nerve blocks can be prolonged by its coadministration with several other compounds, including bupivacaine, lidocaine, ${ }^{14}$ amitriptyline, and others. ${ }^{15}$

Among compounds that have been combined with capsaicin to achieve prolonged duration local anesthesia, site-1 sodium channel blockers, such as tetrodotoxin (TTX), are particularly remarkable for their ability to produce a truly synergistic prolongation of nerve blockade. ${ }^{16}$ Due to their potent local anesthetic effect ${ }^{17,18}$ and minimal local toxicity to muscle and peripheral nerves, ${ }^{18-20}$ site-1 sodium channel blockers are being investigated for clinical use. ${ }^{21}$ While these compounds can cause considerable systemic toxicity at sufficiently large amounts, ${ }^{22}$ this issue can be prevented by their encapsulation in liposomes and other particles, which act as sustained-release systems that slowly release small amounts of drug for long durations of time. ${ }^{23-25}$ The synergy between TTX and capsaicin has been previously demonstrated ${ }^{16}$ and has been attributed to the fact that capsaicin blocks TTX-resistant - but not TTX-sensitive - action potentials in some

sensory nerve fibers, ${ }^{16,26}$ although this mechanism of synergy has not been fully 
elucidated. Interestingly, but for unknown reasons, the prolonged nerve block resulting from this synergy has similar durations of sensory and motor nerve block.

Here, we hypothesized that the combined delivery of capsaicin and TTX by a sustained-release system can achieve prolonged nerve blocks and that selection of the appropriate dose of capsaicin would allow prolonged nerve block to be achieved in the absence of systemic or local toxicity. The latter is particularly important since at higher concentrations, nerve block from capsaicin can last for days or even be irreversible, ${ }^{16}$ likely reflecting neurotoxicity with neuronal atrophy and degeneration; ${ }^{10,27-29}$ additionally, the risk of local toxicity is potentially increased by sustained release, as was the case for bupivacaine. ${ }^{30}$ With these hypotheses in mind, we developed and characterized liposomes containing tetrodotoxin and capsaicin, then tested them in animals to determine their effect on nerve blockade and safety at different concentrations. Finally, we co-injected the two liposome types and studied their combined effect on nerve blockade.

\section{Methods}

\section{Animal care}

Animals were cared for in accordance with protocols approved by the Boston Children's Hospital Institutional Animal Care and Use Committee and the guidelines of the International Association for the Study of Pain. ${ }^{31}$ Adult male Sprague-Dawley rats (Charles River Laboratories, Wilmington, MA) weighing 325-400 g were housed in groups and kept in a 6 am -6 pm light-dark cycle.

\section{Sciatic blockade technique}

Rats were anesthetized with isoflurane-oxygen for $<5$ minutes before receiving injections. A 23-G needle was introduced posteromedially to the greater trochanter, pointing anteromedially, and upon contact with the bone, $0.2 \mathrm{~mL}$ of liposome- or drug-

containing solution was injected. ${ }^{23}$ The left leg was injected and used for blocks, and the 
right leg was not injected. Both legs were tested at each time point, as explained under 'Assessment of nerve blockade'.

\section{Assessment of nerve blockade}

The effectiveness of sensory and motor nerve blocks was measured at the following predetermined time points: $0 \mathrm{~h}$ (before anesthesia), $0.5 \mathrm{~h}, 1 \mathrm{~h}, 2 \mathrm{~h}, 4 \mathrm{~h}, 8 \mathrm{~h}, 12 \mathrm{~h}, 18 \mathrm{~h}, 24 \mathrm{~h}$, 48h, 72h, 96h, 120h, 144h, 168h, 192h, 216h, 240h, 264h, 288h, 312h, 336h. Sensory

nerve block was examined by a modified hotplate test. ${ }^{19,32}$ The plantar surface of the rat's hind paw (in sequence; left, then right) was placed on a hotplate (IITC Inc., Woodland Hills, CA) preheated to $56^{\circ} \mathrm{C}$, and the time until paw withdrawal (termed thermal latency) was recorded. Rats that did not withdraw their paw within 12 seconds were removed from the hotplate to avoid injury or development of hyperalgesia. Each paw was exposed to the hotplate three times at each time point. A median thermal latency above 7 seconds was considered a successful block, since that was the midpoint between maximal latency (12 seconds) and baseline, and nerve block duration was calculated as the time required for median thermal latency to return to 7 seconds from a higher value. Baseline latency has been previously determined by our group to be approximately 2 seconds. Irreversible blocks were defined as blocks not returning to baseline within 14 days of injection.

Motor nerve block was assessed by a weight-bearing test. The animal was positioned over a digital balance allowing it to bear its own weight with one hind paw at a time. The highest weight value the rat could bear without its ankle touching the balance was recorded. A borne weight below its half-maximal value was considered a successful motor block. Motor block duration was calculated as the time required for the borne weight to return halfway to its maximal value. ${ }^{19}$

\section{Solutions of capsaicin and tetrodotoxin}

Tetrodotoxin was dissolved in $20 \mathrm{mM}$ citrate buffer, $\mathrm{pH} 4.45$, to a concentration of $0.3 \mathrm{mM}$. Capsaicin was dissolved in Tween 80 at $55^{\circ} \mathrm{C}$ to a concentration of $330 \mathrm{mM}$. These solutions were stored at $4^{\circ} \mathrm{C}$ and diluted in $\mathrm{NaCl}$ as needed. Capsaicin and TTX solutions were prepared by serial dilution of solutions with known weights/volume 
(mg/ml). In the text, we expressed their concentrations in molarity, so as to facilitate comparison to our previous work and the literature.

\section{Liposome preparation}

i. Capsaicin-loaded liposomes:

Capsaicin liposomes were produced by a modified thin lipid film method. ${ }^{23,24}$ Capsaicin (purity $\geq 95 \%$, Sigma-Aldrich, St. Louis, MO) was mixed with a lipid formulation according to the following molar ratio: 2.4 capsaicin : 1.5 cholesterol (Sigma-Aldrich) : 1.5 DSPC (Avanti Polar Lipids, Alabaster, AL) : 1 DSPG (Genzyme, Cambridge, MA) : 1.2 DLPC (Avanti Polar Lipids). The mixture was dissolved in a solution of 9 chloroform : 1 methanol, and the solvent was then vaporized. The resulting thin lipid film was hydrated with PBS, after which the solution was dialyzed against PBS for $24 \mathrm{~h}$ in a dialysis tube (Cole Parmer, Vernon Hills, IL) with a molecular mass cutoff of $1000 \mathrm{kDa}$.

\section{ii. Tetrodotoxin-loaded liposomes:}

Tetrodotoxin liposomes were also produced using a modified thin lipid film method, as previously reported. ${ }^{23,24} \mathrm{~A}$ lipid formulation at a molar ratio of 3 DSPC : 3 DLPC : 2 DSPG : 3 cholesterol was dissolved in a solution of 9 chloroform : 1 methanol, and vaporized. The solvent was then vaporized, and the resulting thin lipid film was redissolved in tert-butanol, followed by lyophilization. The resulting lipid cake was rehydrated in a solution of $0.3 \mathrm{mg} / \mathrm{mL}$ tetrodotoxin (Abcam, Cambridge, UK) in PBS. After 10 freeze-thaw cycles, the solution was dialyzed against PBS for $24 \mathrm{~h}$ in a dialysis tube with a molecular mass cutoff of $1000 \mathrm{kDa}$.

\section{Liposome characterization}

i. Drug loading:

The concentration of capsaicin loaded into liposomes was determined by highperformance liquid chromatography (Agilent 1260 Infinity, Agilent Technologies, Santa Clara, CA) after disruption of the liposomes with octyl $\beta$-D-glucopyranoside. ${ }^{24,33}$ The 
eluent was monitored at $280 \mathrm{~nm}$ with a flow rate of $1 \mathrm{~mL} / \mathrm{min}$. The concentration of TTX loaded into liposomes was determined by ELISA (Reagen, Moorestown, NJ).

ii. In-vitro drug release:

Solutions of capsaicin-loaded and TTX-loaded liposomes were placed into Slide-ALyzer MINI dialysis devices (Thermo Scientific, Columbia, MD) with a 20,000 MW cutoff. Samples were dialyzed against PBS and incubated at $37^{\circ} \mathrm{C}$ on a platform shaker. At predetermined time points, the dialysate was collected and the dialysis solution was replenished. The concentration of capsaicin was determined by HPLC, and the concentration of TTX was determined by ELISA.

iii. Size measurement:

Capsaicin-loaded and TTX-loaded liposomes were imaged by transmission electron microscopy (TEM) for morphology evaluation and size measurement. $10 \mu \mathrm{L}$ of each liposomal solution was placed on a copper grid, and allowed to dry at room temperature. Each sample was imaged on a Tecnai G2 Spirit BioTWIN transmission electron microscope (FEI, Hillsboro, OR) equipped with an AMT (Woburn, MA) 2k CCD camera, operating at $80 \mathrm{kV}$. Average liposome diameters and their standard deviations were calculated by measuring 100 liposomes of each type using the software ImageJ (NIH). Liposome diameters were also determined by dynamic light scattering (DLS). Solutions of $0.3 \mathrm{~mL}$ of each liposome type were placed into disposable cuvettes (Eppendorf, Hauppauge, NY) and particle sizes were measured using a Delsa Nano C particle analyzer (Beckman Coulter, $\mathrm{CA}$ ) operating at $25^{\circ} \mathrm{C}$.

\section{Tissue harvesting and toxicity studies}

Rats were euthanized with carbon dioxide 4 days after injection at the sciatic nerve. The sciatic nerve and surrounding muscle tissue were dissected for histologic analysis by hematoxylin \& eosin- and toluidine-blue staining, and for analysis by TEM.

The tissues were harvested and processed to produce H\&E-stained slides, using standard techniques. The samples were scored for inflammation (0-4) and myotoxicity (0- 
$6)^{18}$ by a blinded observer. The inflammation score was a subjective assessment of inflammation severity, as follows: $0=$ no inflammation, $1=$ perifascicular inflammation, $2=$ deep inflammation ( $>5$ cell layers), $3=$ hemifascicular inflammation, $4=$ holofascicular inflammation. The myotoxicity score reflected two features characteristic of local anesthetic myotoxicity - nuclear internalization (nuclear localization away from their usual location at the cellular periphery) and regeneration (presence of shrunken myocytes with deeply eosinophilic cytoplasm). Scoring was as follows: $0=$ normal; $1=$ perifascicular nuclear internalization $(<5$ cell layers); $2=$ deep nuclear internalization $(>$ 5 cell layers); $3=$ perifascicular regeneration; $4=$ deep regeneration; $5=$ hemifascicular regeneration; $6=$ holofascicular regeneration.

To evaluate neurotoxicity, the sciatic nerve samples were processed for staining with toluidine blue and TEM, using standard techniques. Toluidine blue staining was done using standard techniques. Briefly, samples were fixed in 1.25\% formaldehyde, $2.5 \%$ glutaraldehyde, and $0.03 \%$ picric acid in $0.1 \mathrm{M}$ sodium cacodylate buffer, $\mathrm{pH} 7.4$, treated with osmium tetroxide, stained with uranyl acetate, dehydrated in graded ethanol solutions, and infiltrated with a mixture of propylene oxide and TAAB 812 Resin (TAAB Laboratories, Calleva Park, UK). Tissues were then cut into $500 \mathrm{~nm}$-thick sections, stained with toluidine blue, and reviewed in a masked fashion with high-resolution light microscopy. ${ }^{24,34}$ The stained nerve sections were evaluated for abnormalities in myelinated nerve fibers (decrease in density, perineurial edema) which included axon abnormalities (size variation, absence) and myelin sheath abnormalities (absence, thickening, splitting).

For transmission electron microscopy, sample fixation and treatment were similar to those for toluidine-blue staining. Samples were cut into thinner, $100 \mathrm{~nm}$-thick sections, and mounted on a metal grid; each section contained $\sim 40 \%$ of a sciatic nerve cross section.

The ultrastructural examination was performed with a JEOL 1200EX transmission electron microscope (JEOL, Tokyo, Japan), equipped with an AMT (Woburn, MA) 2k 
CCD camera, operating at $80 \mathrm{kV}$. For each sciatic nerve sample, 5 micrographs produced at magnifications of $1200 \mathrm{X}$ were used for counting myelinated and unmyelinated fibers, and for measuring fiber diameters. Average fiber diameters were calculated by measuring 100 fibers of each type.

\section{Statistical analysis}

In Figures 1B and 1E, the size of liposomes was depicted as histograms, and the corresponding data in the text were reported as the average diameter and standard deviation calculated by measuring 100 liposomes using the software ImageJ (NIH).

In Figures 2A, 2C, 4A and 4B, binary proportions of animals in each group having reversible or irreversible blocks were compared by Fisher's exact test using a Bonferroni adjustment for as many comparisons as the number of tested concentrations. In Figures 4A and 4B, logistic regression analyses in which TTX concentrations were modeled as categorical variables were used to assess the risk of adverse effects from administering TTX at increasing concentrations, and corresponding Wald tests (distributed as chisquare statistics) were reported in the text. ${ }^{35}$

In Figures $2 \mathrm{~B}$ and $4 \mathrm{C}$, our test of normality indicated significant departure from normality, hence we applied the non-parametric Mann-Whitney-U test with Bonferroni adjustment for multiple comparisons, and report the corresponding data as medians and IQRs. In Figure 5, where such departure from normality was not noted, nerve block data were reported as means and standard deviation [denoted throughout as mean (SD)] and were assessed using ANOVA with Bonferroni correction for multiple comparisons. ${ }^{36}$ In Figure 6, we used the F-test in ANOVA with Bonferroni adjustment for multiple comparisons.

In table 2, tissue reaction data are reported as median and IQR and compared between groups using the non-parametric Kruskal-Wallis test using a Bonferroni adjustment to protect against type I errors. ${ }^{37}$ Since they did not depart significantly from a normal Gaussian-shaped distribution, as evaluated by the Shapiro-Wilk test, percentage and 
diameter of unmyelinated fibers are presented as mean and standard deviation with groups compared using ANOVA with Bonferroni adjustment for multiple comparisons.

Statistical analysis was performed using IBM SPSS Statistics. All reported p-values are two-sided. All analyses underwent Bonferroni adjustment for the number of multiple comparisons within each figure panel, to control for type I errors with an alpha level of 0.05 .

For our animal studies, we chose a sample size of $n=4$ per group to give us at least $80 \%$ statistical power to detect differences in sensory block duration based on an large expected effect size $>2$ (e.g. $\sim 8$ hour difference in mean sensory block duration between our two longest-lasting formulations, each with a variability (standard deviation) of $\sim 2-3$ hours, hence an expected effect size, or Cohen's d, of $\sim 2.67$ ). 


\section{Results}

\section{Liposome production and characterization}

Capsaicin liposomes were produced by a modified thin lipid film method ${ }^{23,24}$ (Table 1). TEM demonstrated that the liposomes were spherical (Fig. 1A) and had an average diameter of $1.29(0.41) \mu \mathrm{m}$ (Fig. 1B). Measurements by DLS revealed an average diameter of $1.42(0.33) \mu \mathrm{m}$. The liposomal encapsulation of capsaicin resulted in prolonged release of capsaicin compared to that of free capsaicin (Fig. 1C).

Tetrodotoxin liposomes were also produced using a modified thin lipid film method $^{23,24}$ (Table 1). Similarly to the capsaicin-loaded liposomes, tetrodotoxin-loaded liposomes were roughly spherical (Fig. 1D) and had an average diameter of $1.22(0.38)$ $\mu \mathrm{m}$ (Fig.1E), measured by TEM; by DLS, their diameter was $1.36(0.40) \mu \mathrm{m}$. Liposomal encapsulation resulted in prolonged release of tetrodotoxin (Fig. 1F).

\section{Capsaicin liposomes}

\section{TTX liposomes}

$\begin{array}{ccc}\text { Theoretical drug loading a } & 30 \%(\mathrm{w} / \mathrm{w}) & 1 \%(\mathrm{w} / \mathrm{w}) \\ \text { Measured drug loading }{ }^{\mathrm{b}} & 12.1 \pm 4.2 \%(\mathrm{w} / \mathrm{w}) & 0.13 \pm 0.05 \%(\mathrm{w} / \mathrm{w}) \\ & & \\ \text { Encapsulation efficiency }{ }^{\mathrm{c}} & 40.34 \pm 14.04 \%(\mathrm{w} / \mathrm{w}) & 13.4 \pm 5.3 \%(\mathrm{w} / \mathrm{w}) \\ & 1.5 \text { cholesterol : } 1.5 \mathrm{DSPC}: 1 & 1.5 \text { cholesterol }: 1.5 \mathrm{DSPC}: 1 \\ \text { Lipid composition } & \text { DSPG }: 1.2 \mathrm{DLPC} & \text { DSPG : } 1.5 \mathrm{DLPC} \\ & 1.29 \pm 0.41 \mu \mathrm{m} & 1.22 \pm 0.38 \mu \mathrm{m}\end{array}$

Table 1. Characterization of liposomes. ${ }^{\text {a }}$ Mass of added drug / (mass of added drug + mass of added lipid) x $100 .{ }^{b}$ Measured mass of loaded drug / (mass of added drug + mass of added lipid) x 100. ${ }^{\mathrm{c}}$ Measured drug loading / Theoretical drug loading x 100. Data are means $\pm \mathrm{SD} ; \mathrm{n}=3$. TTX: tetrodotoxin, DSPC: 1,2-distearoyl-sn-glycero-3phosphocholine, DSPG: 1,2-distearoyl-sn-glycero-3-phospho-(1'-rac-glycerol), DLPC: 1,2-dilinoleoyl-sn-glycero-3-phosphocholine. 


\section{Capsaicin liposomes}
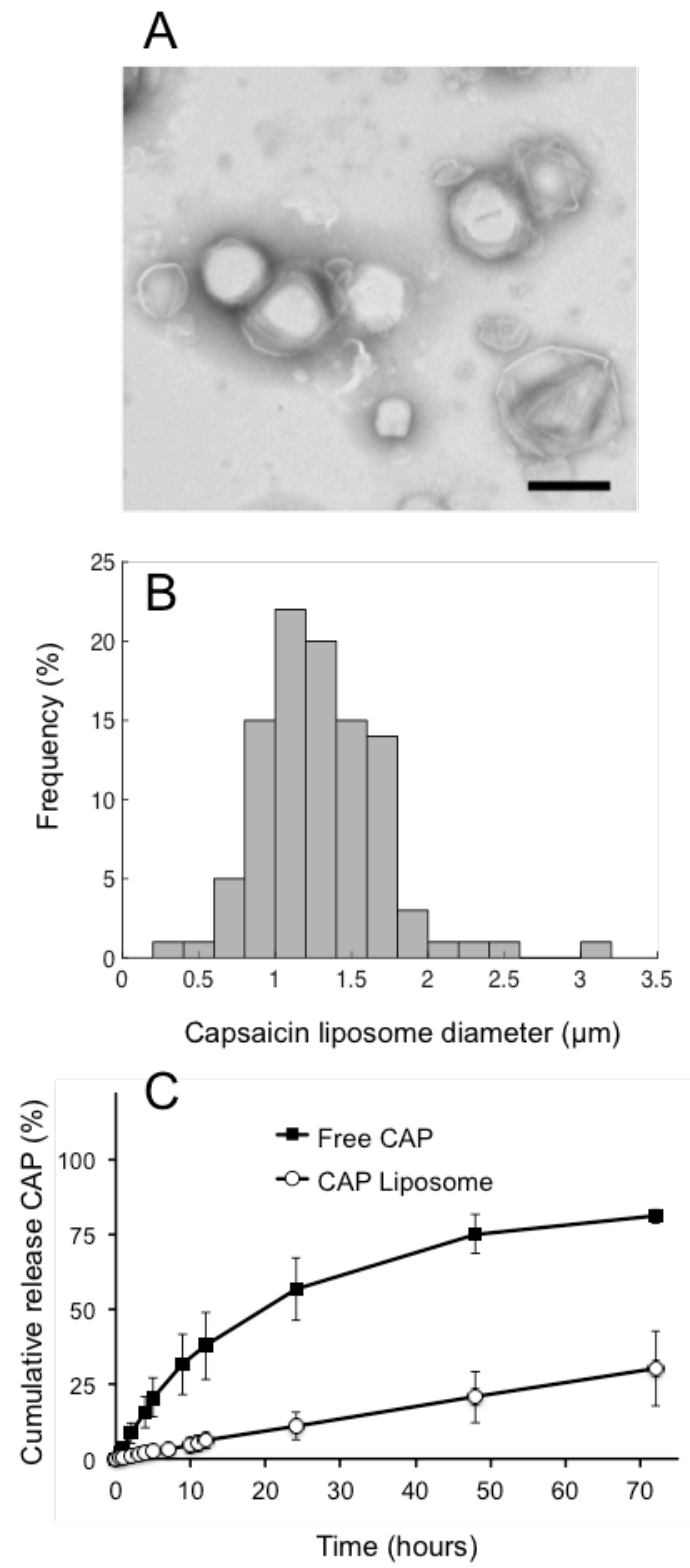

TTX liposomes
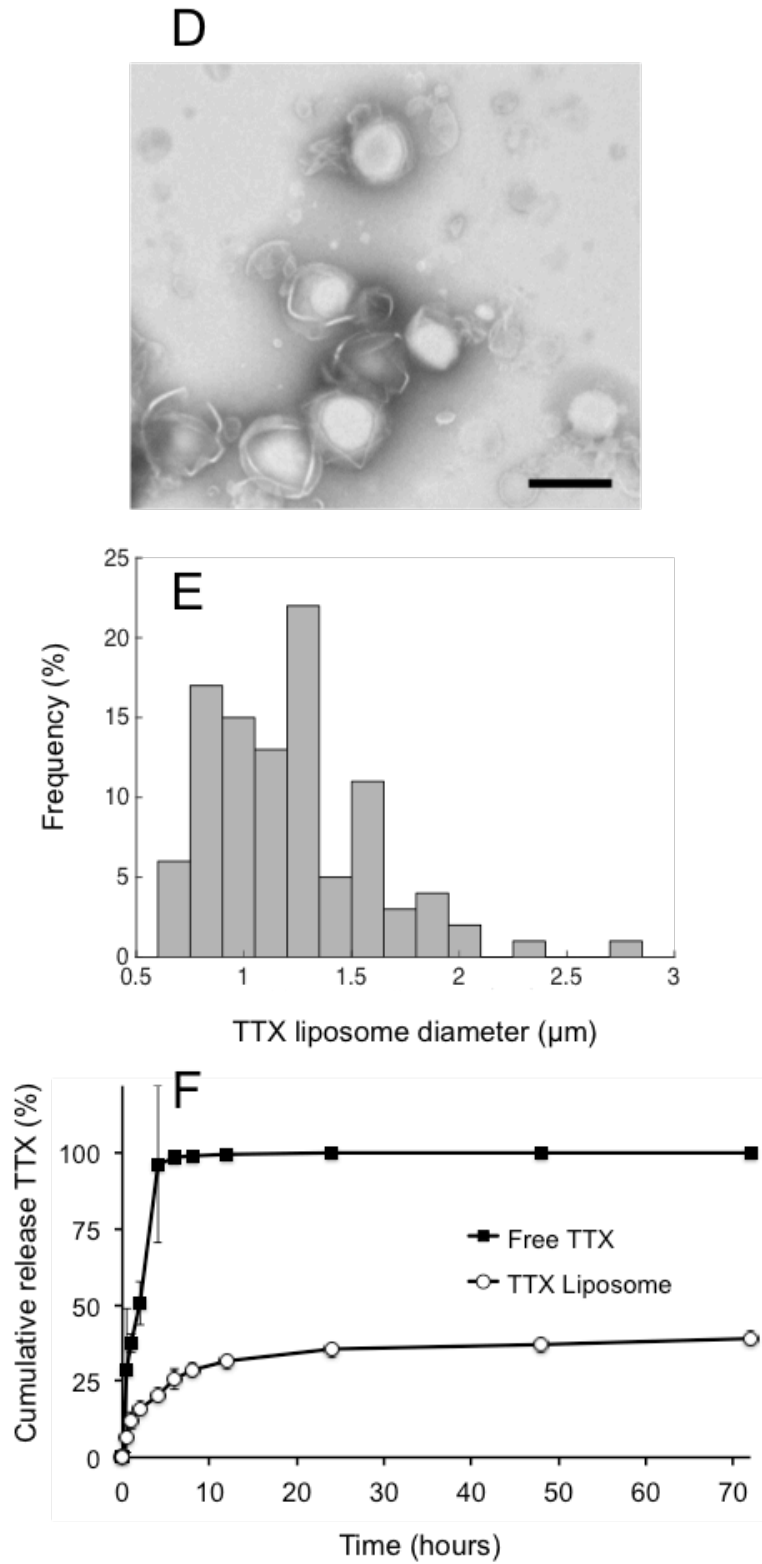

Figure 1. Characterization of capsaicin and TTX liposomes. (A, D) Representative transmission electron micrographs of capsaicin and TTX liposomes; scale bars are $1 \mu \mathrm{m}$. (B, E) Size distribution of 100 capsaicin and TTX liposomes as measured from TEM. (C, F) Cumulative release of capsaicin and TTX from liposomes in vitro, as percentages of total encapsulated drug. Data in $(\mathrm{C})$ and $(\mathrm{F})$ are means $\pm \mathrm{SD}, \mathrm{n}=3$ per group. CAP: capsaicin; TTX: tetrodotoxin. 


\section{Sciatic nerve block by free and liposomal capsaicin}

A key design feature was to encapsulate capsaicin at the highest loading that would not cause nerve injury, to maximize the possible safe interaction with tetrodotoxin. To this end, we performed sciatic nerve injections in rats with $0.2 \mathrm{~mL}$ of either capsaicinloaded liposomes or free capsaicin, followed by neurobehavioral testing (Fig. 2). Testing was done at $819 \mu \mathrm{M}, 1637 \mu \mathrm{M}, 3274 \mu \mathrm{M}$ capsaicin, representing serial dilutions of capsaicin (in mass/volume, these were 250, 500, and $1000 \mu \mathrm{g} / \mathrm{mL}$, respectively). Four animals were used for each experimental group.
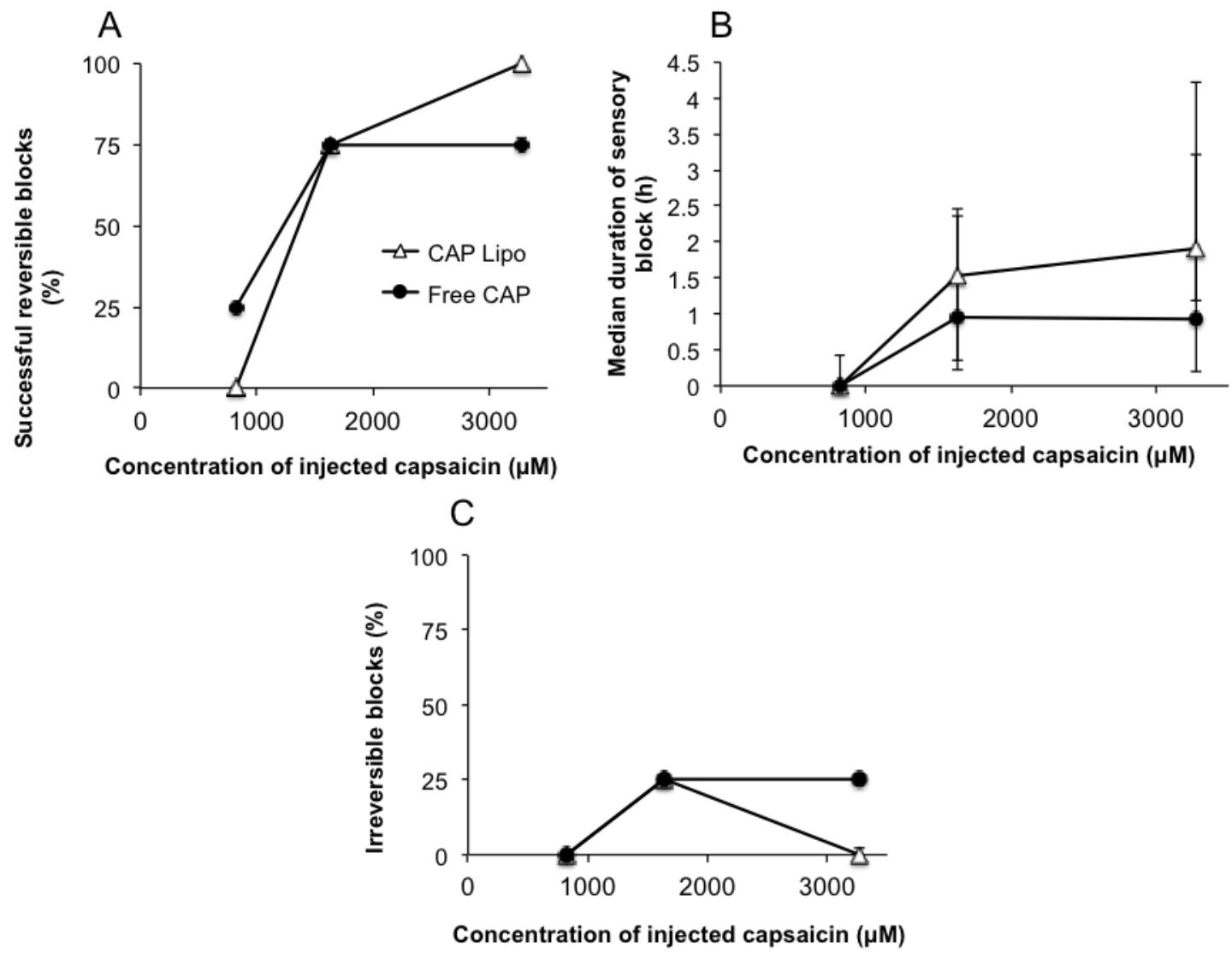

Figure 2. In vivo sensory nerve blockade with free or liposomal capsaicin. (A) Percentage of animals with a successful block. (B) Median duration of reversible blocks. (C) Percentage of animals with an irreversible block. Data in (B) are medians with $25^{\text {th }}$ and $75^{\text {th }}$ percentiles; $n=4$ per group per concentration. Lipo: liposomes, CAP: capsaicin. 
There were no statistically significant differences between capsaicin liposomes and the equivalent dose of free capsaicin in the incidence of successful reversible or irreversible nerve blocks (Fig. 2A and 2C; P > 0.99, Fisher's exact test), or in the median duration of nerve blocks (Fig. 2B; $\mathrm{P}=0.69$ for $819 \mu \mathrm{M}, \mathrm{P}=0.68$ for $1637 \mu \mathrm{M}$, and $\mathrm{P}=$ 0.20 for $3274 \mu \mathrm{M}$, Mann-Whitney $\mathrm{U}$ test). There were no motor deficits, and no animals died or exhibited contralateral deficits (right paw thermal latency above $7 \mathrm{~s}$, which would indicate systemic drug distribution). As previously shown, irreversible blocks from injection of high amounts of capsaicin were characterized by an initial transient increase in thermal latency, followed by some degree of block resolution, then by a very longlasting increase in latency (Fig. 3). Additionally, no animals were observed to have any behavioral abnormalities after injection of pure perineural capsaicin, aside from thermal latency changes: they did not appear uncomfortable, and were noted to feed, breathe and walk normally.

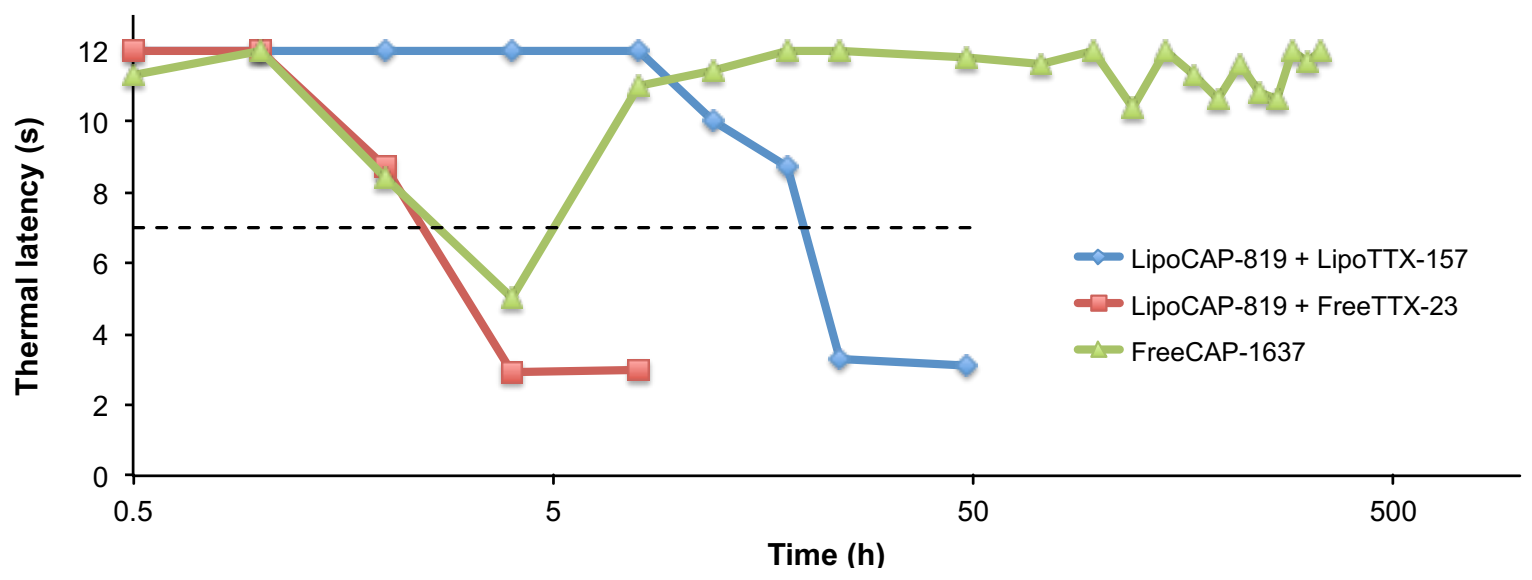

Figure 3. Illustrative time courses of thermal latency. Animals injected with LipoCAP819 + FreeTTX-23 or LipoCAP-819 + LipoTTX-157 had a transient (reversible) increase in thermal latency. Some animals injected with high concentrations of capsaicin (in this case, FreeCAP-1637) had an initial transient increase in latency, followed (with varying degrees of intervening resolution) by a very long-lasting (irreversible) increase in latency. Note that the $\mathrm{x}$-axis is in log scale. 
The highest concentration of liposomal capsaicin tested that did not result in any irreversible blocks was $819 \mu \mathrm{M}$ capsaicin. While that concentration resulted in minimal nerve blocks, we hypothesized that it would provide synergy to prolong the duration of block from TTX liposomes. Therefore, all subsequent experiments were conducted using $819 \mu \mathrm{M}$ capsaicin liposomes, termed LipoCAP-819.

\section{Sciatic nerve block by free and liposomal tetrodotoxin}

Another key design feature was to encapsulate tetrodotoxin at the highest loading that would not cause systemic toxicity, in order to maximize the possible safe interaction with capsaicin. We performed sciatic nerve injections in rats with either TTX-loaded liposomes or free TTX, followed by neurobehavioral testing. Four animals were used for each experimental group (Fig. 4).

Free TTX produced nerve blocks in a concentration-dependent manner, which is well established $^{19,38,39}$, until $47 \mu \mathrm{M}$, above which most animals died (Fig. 4A). The risk of adverse effects or death was noted to increase significantly with increases in free TTX concentration (Wald test $=24.1, \mathrm{P}<0.001$ comparing $73 \mu \mathrm{M}$ with all other concentrations). The highest concentration of free TTX that did not result in adverse effects (deficits in the contralateral leg or death) was $23 \mu \mathrm{M}$ (Fig. 4A), termed FreeTTX23. This concentration produced a sensory block with a mean duration of 11 (23) minutes. 

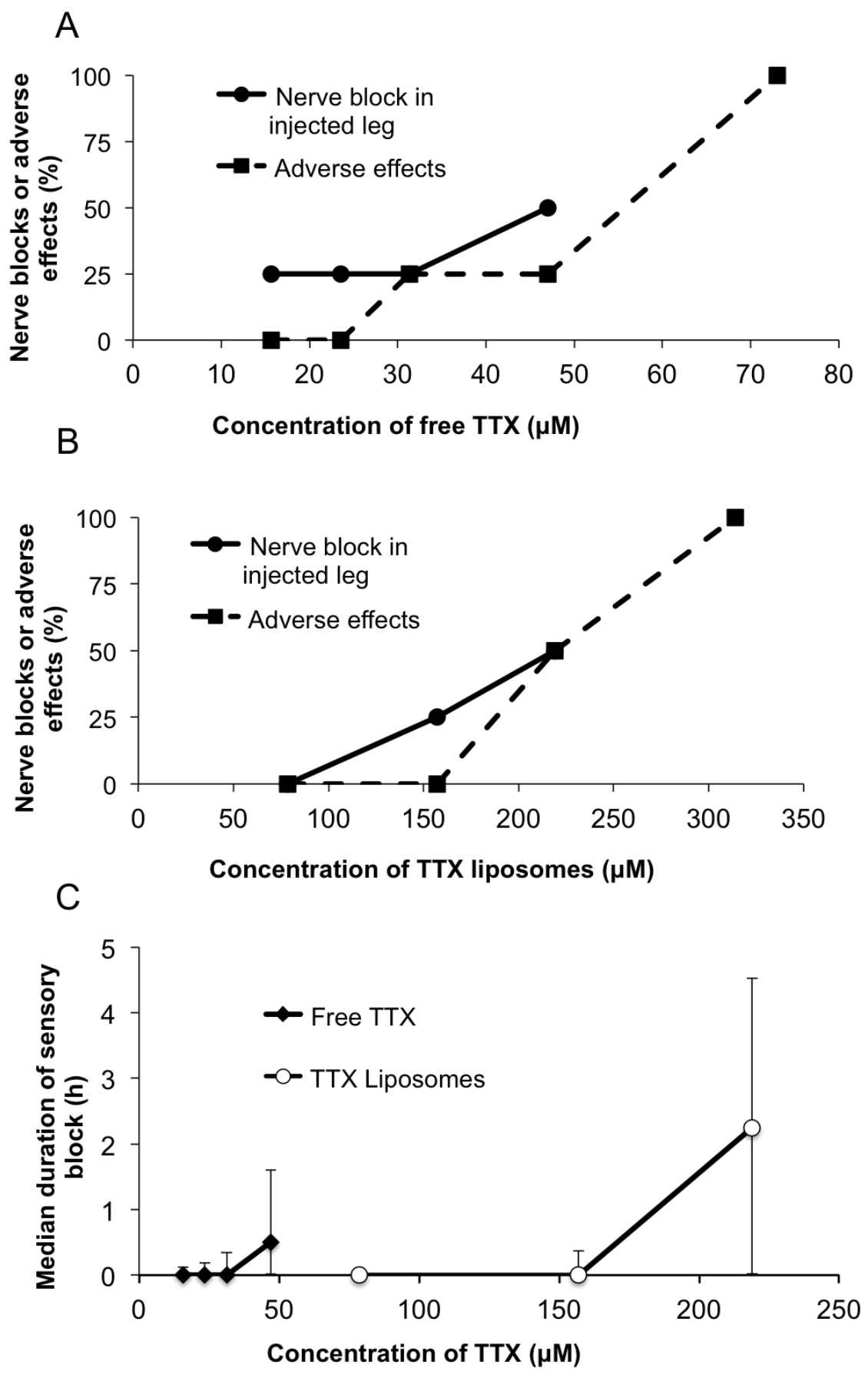

Figure 4. In vivo sensory nerve blockade with free or liposomal TTX. (A) Percentage of animals with a successful nerve block or adverse effects (block in the uninjected extremity and/or death) from free TTX. (B) Percentage of animals with a successful nerve block or adverse effects from TTX liposomes. (C) Median duration of sensory blocks from free and encapsulated TTX. Data in (C) are medians with $25^{\text {th }}$ and $75^{\text {th }}$ percentiles; $n=4$ per group per concentration. TTX: tetrodotoxin. 
Similarly to free TTX, increasing concentrations of TTX liposomes resulted in an increased risk of adverse effects or death (Wald test $=874.2, \mathrm{P}<0.001$ ), with $314 \mu \mathrm{M}$ resulting in significantly higher percentages of those effects than lower concentrations $(\mathrm{P}$ $<0.001$ compared to $78.5 \mu \mathrm{M}, \mathrm{P}<0.001$ compared to $157 \mu \mathrm{M}, \mathrm{P}=0.046$ compared to $219 \mu \mathrm{M})$. The highest concentration of TTX liposomes that did not result in adverse effects was $157 \mu \mathrm{M}$ TTX liposomes (Fig. 4B), termed LipoTTX-157. This concentration produced a sensory block with mean duration of 22 (44) minutes. The tested concentrations of TTX liposomes and free TTX did not overlap (since encapsulation allows safe delivery of much more TTX, but also requires greater loading for therapeutic effect, as seen here). TTX liposomes were able to produce longer nerve block durations.

\section{Sensory nerve block from tetrodotoxin liposomes co-injected with capsaicin}

\section{liposomes}

Having established the upper limits of safe dosing (i.e. without detectable adverse effects given our sample size) of the two liposome types, we tested them in combination. In order to maintain constant the volume of injectate $(0.2 \mathrm{~mL})$, the mass of lipid, and the concentrations of active compounds, LipoCAP-819 and LipoTTX-157 were combined by co-injecting $0.1 \mathrm{~mL}$ of each. When they were injected separately, they were delivered in $0.1 \mathrm{~mL}$ together with $0.1 \mathrm{~mL}$ of blank liposomes. Four animals were used for each experimental group (Fig. 5). Both single-compound injections caused minimal nerve block, while co-injection of LipoCAP-819 and LipoTTX-157 caused nerve block lasting 18.2 (3.8) hours $(\mathrm{P}<0.001$, ANOVA, $\mathrm{n}=4$ per group) (Fig. $5 \mathrm{~A})$. Animals were also injected with $0.2 \mathrm{~mL}$ of LipoCAP-819 or LipoTTX-157. Both of these resulted in shorter sensory blocks than the combination injection $(\mathrm{P}<0.001$ for both, ANOVA, $\mathrm{n}=4$ per group). Injection of $0.2 \mathrm{~mL}$ of $157 \mu \mathrm{M}$ TTX liposomes resulted in contralateral block in 1 of 4 animals. No other animals had any adverse effects.

\section{Functional specificity of liposomal types}

While injection of capsaicin-only formulations resulted in purely sensory blocks, formulations containing tetrodotoxin resulted in some degree of motor blockade. The 
combination of $0.1 \mathrm{~mL}$ LipoCAP-819 with $0.1 \mathrm{~mL}$ LipoTTX-157 resulted in a motor block lasting 12.6 (4.9) hours, which was longer than that from both types of singlecompound injections ( $\mathrm{P}<0.001$, ANOVA, $\mathrm{n}=4$ per group) (Fig. 5B). There was no statistically significant difference between the durations of sensory and motor block in any of the tetrodotoxin-containing formulations. 

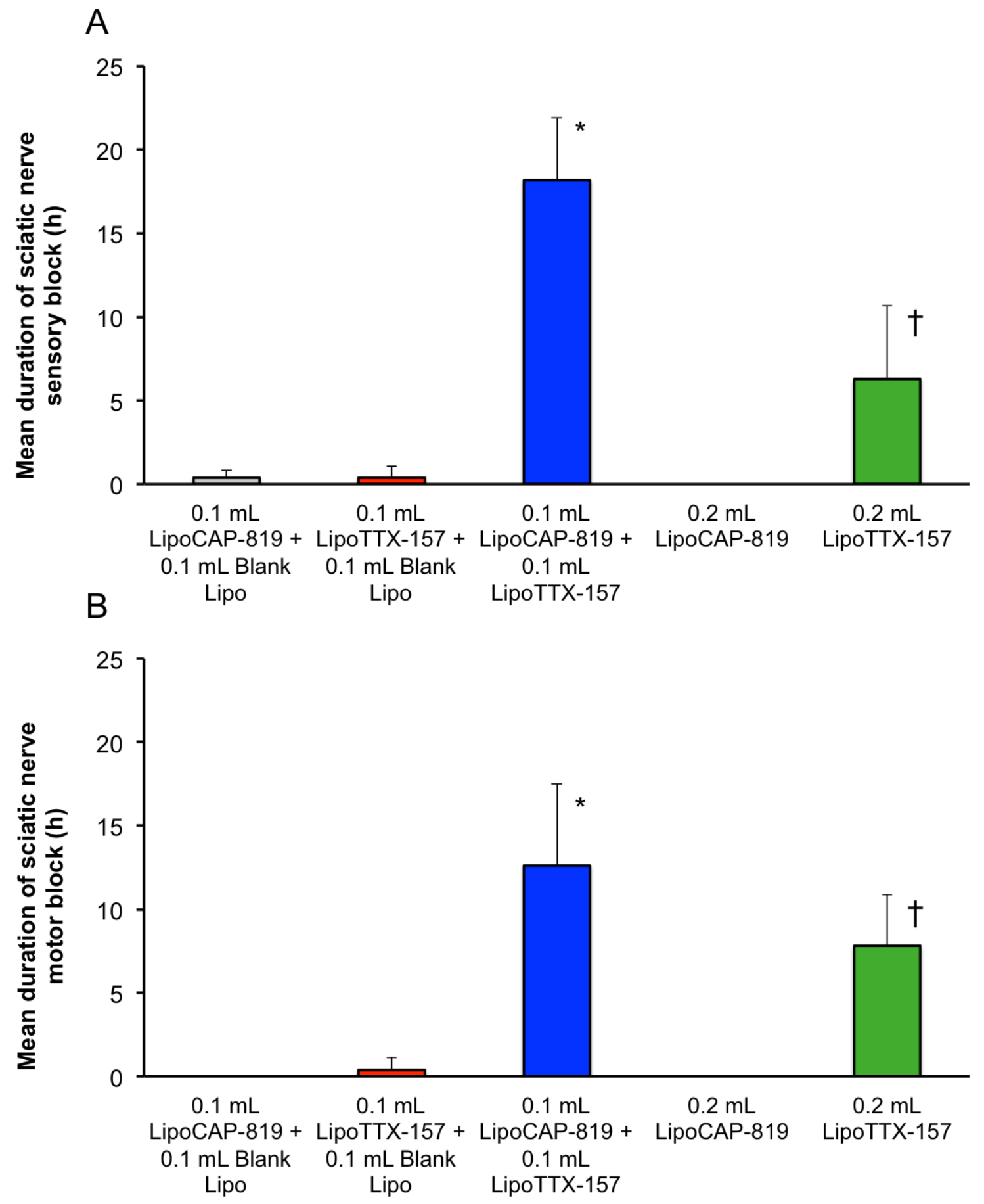

Figure 5. Sciatic nerve sensory (A) and motor (B) blockade from co-injection of tetrodotoxin liposomes and capsaicin liposomes. Data are means with standard deviations, $\mathrm{n}=4$ per group. $* \mathrm{P}<0.001$ vs. each of the other groups, ANOVA. $\dagger 0.2 \mathrm{~mL}$ LipoTTX-157 vs. $0.2 \mathrm{~mL}$ LipoCAP-819, with $\mathrm{P}=0.038$ for sensory (A) and $\mathrm{P}=0.006$ for motor (B) block. LipoCAP-819: liposomes with $819 \mu \mathrm{M}$ capsaicin. LipoTTX-157: liposomes with $157 \mu \mathrm{M}$ tetrodotoxin. 


\section{Effect of free drugs on nerve block from liposome-encapsulated drugs}

To demonstrate that encapsulation was important to the effect achieved by the liposome combination, we injected LipoCAP-819 with the highest concentration of free TTX that did not result in systemic toxicity (Fig. 4A), $23 \mu \mathrm{M}$ (FreeTTX-23), which alone provided a mean duration of nerve block of 11 (23) minutes with no contralateral deficits (Fig. 6A). The combination achieved a mean duration of sensory block of 177 (66) minutes, which is much less than that from LipoCAP-819 combined with LipoTTX-157 $(\mathrm{P}=0.004$, F-test in ANOVA, $\mathrm{n}=4)$.

LipoTTX-157 were then injected with the highest concentration of free capsaicin that did not result in irreversible block (Fig. 2C), $819 \mu \mathrm{M}$ (termed FreeCAP-819), which alone provided a mean duration of nerve block of 8 (17) minutes (Fig. 6B). The combination achieved a mean duration of sensory block of 207 (278) minutes, which was also less than that from LipoCAP-819 combined with LipoTTX-157 ( $\mathrm{P}=0.012$, F-test in ANOVA, $n=4)$.

These data showed that co-delivery of encapsulated drugs yielded longer nerve blockade than did delivery of single drugs (encapsulated or not) or single encapsulated drugs with the second drug free. 

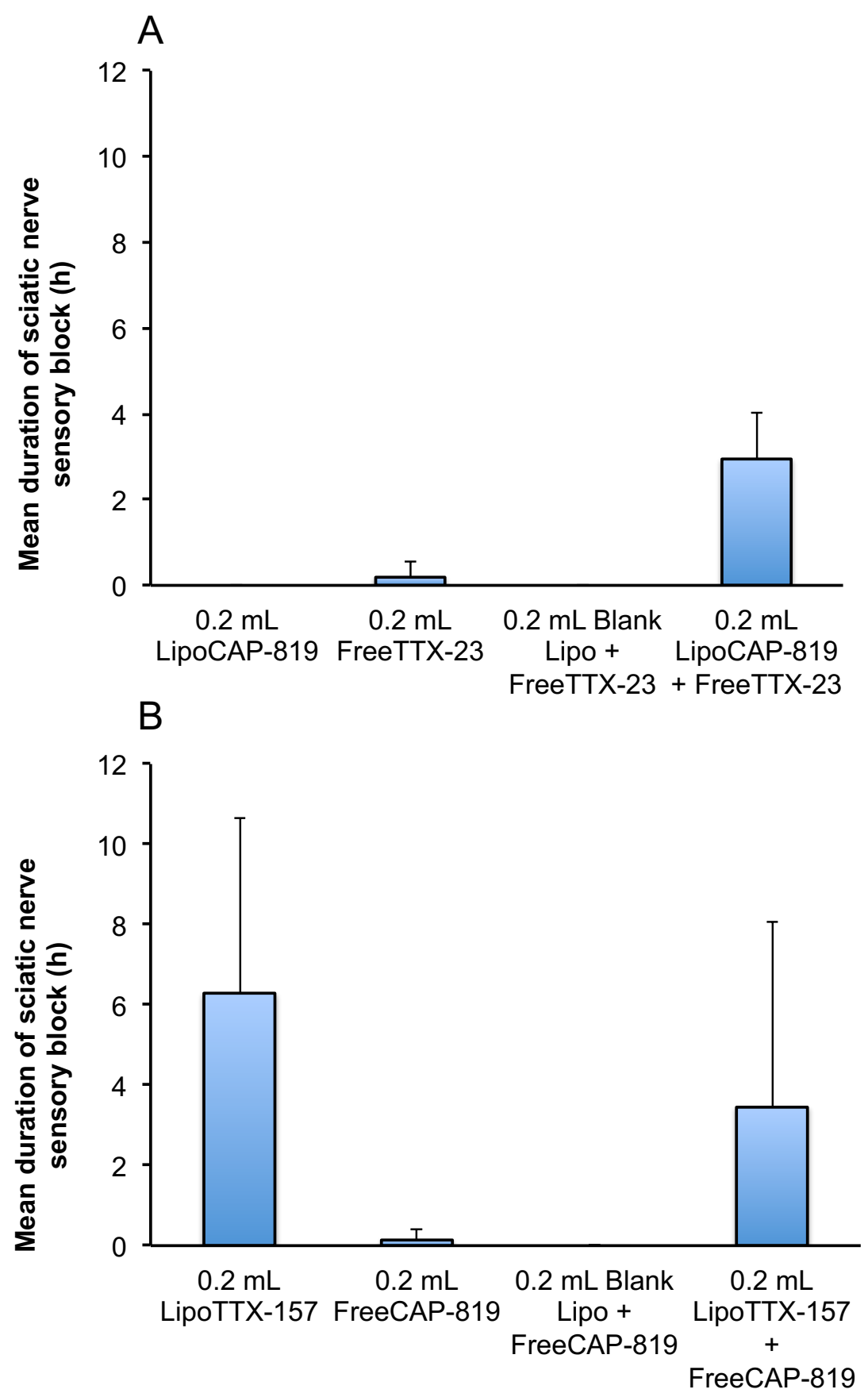

Figure 6. Sciatic nerve sensory blockade from co-injection of liposome-encapsulated drugs and free drugs. (A) Co-injection of capsaicin liposomes and free TTX. (B) Coinjection of TTX liposomes and free capsaicin. Data are means with standard deviations; $\mathrm{n}=4$ per group. LipoCAP-819: liposomes with $819 \mu \mathrm{M}$ capsaicin, FreeTTX-23: $23 \mu \mathrm{M}$ free tetrodotoxin, LipoTTX-157: liposomes with $157 \mu \mathrm{M}$ tetrodotoxin, FreeCAP-819: $819 \mu \mathrm{M}$ free capsaicin. 


\section{Tissue reaction}

The sciatic nerves and adjacent tissues of all animals were harvested 4 days after injection. Hematoxylin-eosin stained sections were prepared and scored for myotoxicity and inflammation. ${ }^{39,40}$ Two animals that received the liposome combination showed scattered lymphocytes and mild nuclear internalization within myocytes (Fig. 7A); the rest of the animals in that group showed no inflammation or myotoxicity, similarly to uninjected animals (Fig. 7B). Animals injected with that combination had median inflammation and myotoxicity scores of 0.5 (ranges [0-1] and [0-2], respectively), with no statistically significant differences compared to uninjected animals (Table 2). The combination of FreeTTX-23 and LipoCAP-819 revealed no apparent differences in histology and had low scores for inflammation [median 0 (range 0-1)] and myotoxicity [0 (0-1)], and so did the combination of FreeCAP-819 and LipoTTX-157 [inflammation 0 $(0-1)$ and myotoxicity $0(0-0)]$.

Sciatic nerve segments were also processed with toluidine-blue staining ${ }^{34,41}$ for more sensitive detection of potential neurotoxicity to myelinated nerve fibers. Nerve histology from animals co-injected with LipoCAP-819 and LipoTTX-157 showed no significant differences when compared to uninjected animals or any other groups in terms of axonal size, shape, distribution, and myelin thickness (Fig. 7C and D). 


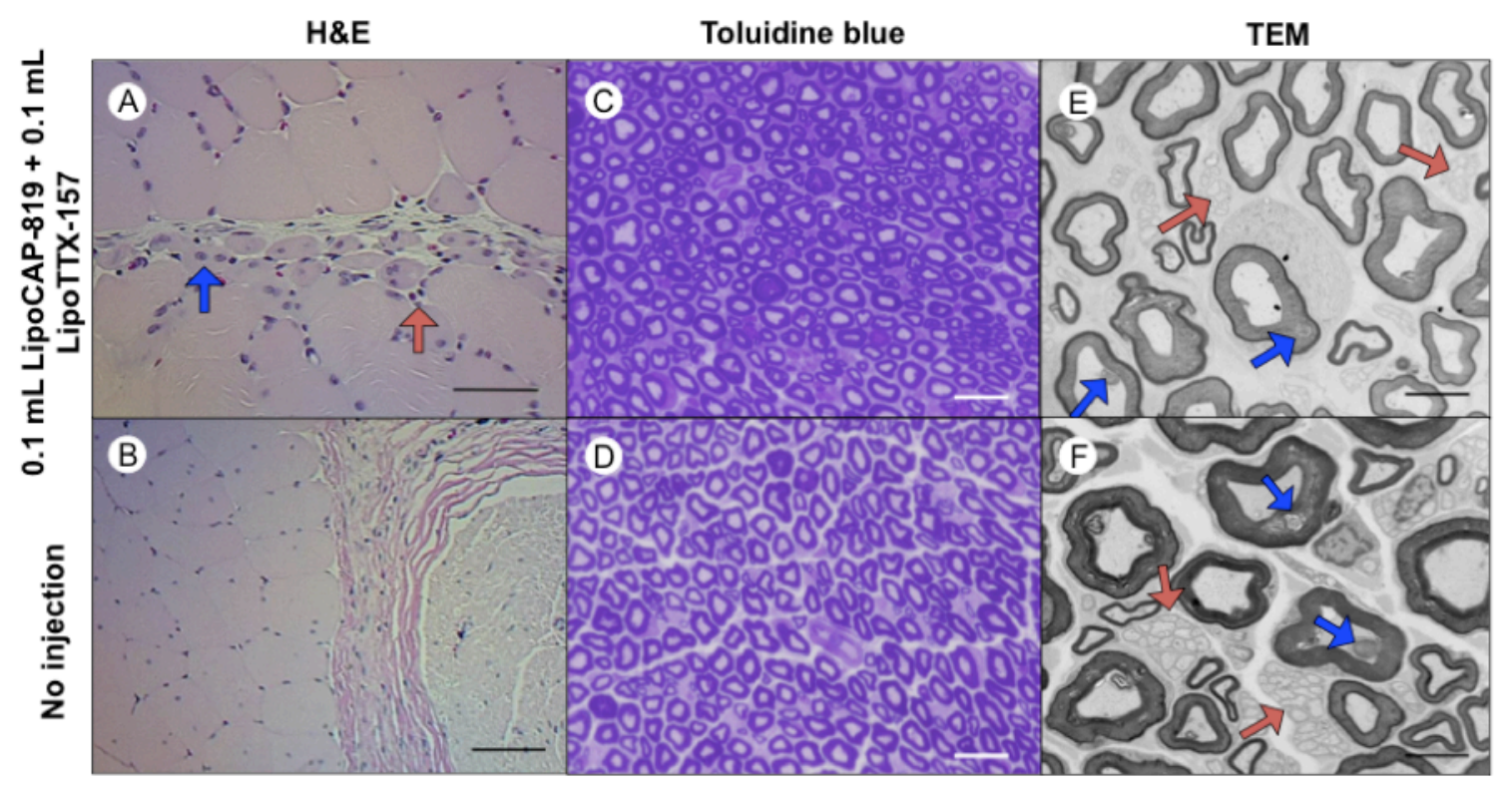

Figure 7. Representative histology and transmission electron microscopy of dissected sciatic nerves and surrounding muscle tissue. Top row $(\mathrm{A}, \mathrm{C}, \mathrm{E})$ : injection of $0.1 \mathrm{~mL}$ LipoCAP-819+0.1 mL LipoTTX-157. Bottom row (B, D, F): no injection. (A,B) H\&Estained sections of muscle adjacent to injection site; red arrow denotes scattered lymphocytes, blue arrow denotes perifascicular internalization of nucleus. (C, D) Toluidine blue-stained sciatic nerve sections. (E,F) Transmission electron micrographs of sciatic nerve sections; red arrows denote unmyelinated fibers, blue arrows denote deposits within axons or myelin sheath. Scale bars $50 \mu \mathrm{m}$ (A), $100 \mu \mathrm{m}$ (B), $20 \mu \mathrm{m}(\mathrm{C}, \mathrm{D})$, $5 \mu \mathrm{m}$ (E,F). LipoCAP-819: liposomes with $819 \mu \mathrm{M}$ capsaicin, LipoTTX-157: liposomes with $157 \mu \mathrm{M}$ tetrodotoxin, $\mathrm{H} \& \mathrm{E}$ : haematoxylin and eosin, TEM: transmission electron microscopy.

Since TRPV1 agonists have been reported to cause damage primarily to unmyelinated fibers ${ }^{28}$ resulting in a decrease in their number ${ }^{27}$ and diameter, ${ }^{42}$ we also examined all sciatic nerves by TEM, a technique that enables clear visualization of both myelinated and unmyelinated fibers. ${ }^{27,42}$ Electron micrographs obtained from animals co-injected with LipoCAP-819 and LipoTTX-157 showed normal unmyelinated fibers, with no evidence of injury, such as swelling, disintegration, or dark-staining axoplasm. ${ }^{42}$ In both treated and untreated groups, myelinated fibers were occasionally found to have deposits within axons or circular breaks within myelin sheaths (Fig. 7E and F), which are commonly reported electron-microscopic findings of normal peripheral nerve fibers. ${ }^{42,43}$ We calculated the percentage of unmyelinated fibers in sciatic nerve segments from animals that received the liposome combination and animals that were not injected, and 
quantified the average diameter of such fibers (Table 2). There was no statistically significant difference in the percentage or diameter of unmyelinated fibers between these two groups ( $\mathrm{P}>0.05$ for both, ANOVA). To provide a positive control for nerve injury from capsaicin, we injected a group of animals with $0.2 \mathrm{~mL}$ of $16.4 \mathrm{mM}(1 \mathrm{mg})$ capsaicin - the equivalent of 40 times the mass of capsaicin delivered via the liposome combination - which has been shown to cause a reduction in unmyelinated fibers of up to $30 \%{ }^{27}$ This group had a percentage of unmyelinated fibers of $54.2(4.3) \%$, and an average unmyelinated fiber diameter of $0.9(0.2) \mu \mathrm{m}$, representing a statistically significant reduction in the percentage $(\mathrm{P}<0.001$, ANOVA), but not in the average fiber diameter ( $\mathrm{P}$ $=0.151$, ANOVA) of unmyelinated fibers when compared to the untreated controls. 
Percentage of Unmyelinated

\begin{tabular}{|c|c|c|c|c|}
\hline Group & $\begin{array}{c}\text { Inflammation } \\
\text { score }\end{array}$ & $\begin{array}{c}\text { Myotoxicity } \\
\text { score }\end{array}$ & $\begin{array}{c}\text { unmyelinated } \\
\text { fibers } \\
(\%)\end{array}$ & $\begin{array}{c}\text { fiber } \\
\text { diameter } \\
(\mu \mathrm{m})\end{array}$ \\
\hline No injection & $0(0-0)$ & $0(0-0)$ & $72(2)$ & $1.02(0.44)$ \\
\hline LipoCAP-819+ & $0.5(0-1)$ & $0.5(0-2)$ & $73(3)$ & $0.97(0.23)$ \\
\hline \multicolumn{5}{|l|}{ LipoTTX-157 } \\
\hline $16.4 \mathrm{mM}$ capsaicin & $1.5(0-2)$ & $1.5(0-2)$ & $54(4)^{*}$ & $0.94(0.24)$ \\
\hline $\mathrm{P}$ value $\dagger$ & 0.087 & 0.119 & $<0.001$ & 0.151 \\
\hline
\end{tabular}

Table 2. Scoring of hematoxylin-eosin images for inflammation, myotoxicity, and quantification of unmyelinated fiber parameters from transmission electron microscopy. Inflammation score range: $0-4$; myotoxicity score range: $0-6$. Data for inflammation and myotoxicity are medians and range. Data for percentage and diameter of unmyelinated fibers are mean (SD)

$\uparrow$ Comparison of data for inflammation and myotoxicity by the nonparametric KruskalWallis test; $\mathrm{n}=4$ animals per group. Data for percentage and diameter of unmyelinated fibers are compared using ANOVA, $n>400$ fibers for percentage calculation, $n=100$ fibers for diameter measurement.

$* \mathrm{P}<0.001$ versus no injection and LipoCAP-819 + LipoTTX-157, ANOVA with Bonferroni correction. LipoCAP-819: liposomes with $819 \mu \mathrm{M}$ capsaicin, LipoTTX-157: liposomes with $157 \mu \mathrm{M}$ tetrodotoxin. 


\section{Discussion}

The co-injection of capsaicin liposomes and TTX liposomes led to a marked prolongation of nerve block, with blocks lasting approximately 18 hours. By way of comparison, in this same animal model, sensory block from $0.5 \%$ bupivacaine $(\mathrm{w} / \mathrm{v})$ solution lasts approximately $2-2.5 \mathrm{~h},{ }^{19}$ and that from liposomal $1.33 \%$ bupivacaine lasts approximately 4 hours. ${ }^{34}$ This block prolongation was greater than that achieved by capsaicin liposomes co-injected with free TTX or TTX liposomes co-injected with free capsaicin, demonstrating that encapsulation of both drugs was necessary for the observed effect. Additionally, tissue reaction to the combination of liposomes was benign. The percentage of unmyelinated fibers in our treated animals was no different from that of our non-injected control animals, which was consistent with existing literature for the normal rat sciatic nerve. ${ }^{44}$ Importantly, neurotoxicity due to capsaicin, which has been widely reported, ${ }^{27-29,45,46}$ was not seen. Given that TRPV1 agonists may induce neuronal degeneration as early as one day after their administration, ${ }^{47,48}$ the timing of our animal dissections (4 days) should have allowed detection of such degeneration. While capsaicin in solution has been commonly used for local anesthesia, including in combination with site-1 sodium channel blockers, ${ }^{14-16}$ to our knowledge, this is the first time that capsaicin liposomes have been used for this purpose. Likewise, this is the first study in which a liposomal formulation achieving a significantly prolonged nerve block was shown to have no detectable toxicity by H\&E, toluidine-blue staining and quantification of nerve fibers by TEM.

This study was partially inspired by previous work from our group demonstrating synergy between free capsaicin and free TTX in sciatic nerve blockade. ${ }^{16}$ The exact mechanism of this synergy is not clear, but it is possible that capsaicin and TTX potentiate each other because they inhibit different populations of sodium currents, mediated by TTX-resistant and TTX-sensitive channels, respectively. In fact, Großkreutz et al. and Yoshimura et al., among others, have shown that capsaicin can block TTXresistant sodium potentials in C fibers, ${ }^{26,49}$ and Kwong and Lee showed that capsaicinsensitive pulmonary neurons have a higher percentage of TTX-resistant sodium currents 
than do capsaicin-insensitive pulmonary neurons. ${ }^{50}$ When combined with conventional local anesthetics, capsaicin has been shown to prolong sensory-specific nerve blocks, by other groups. ${ }^{14,15}$ However, similarly to our previous findings on the combination of capsaicin and TTX, we found no significant predominance of either sensory or motor blockade in any of the studied groups in our current work.

This work was oriented toward studying the effect of formulation on nerve block duration. Another important metric is the onset of nerve blockade. We did not see any difference between groups in that metric, since the hind paw thermal latency was above $7 \mathrm{~s}$ in all animals that developed blockade by the first tested time point. However, since that time point was 30 minutes after injection, it is possible that there were differences in onset that were not detected. For example, differences in onset between various site-1 sodium channel blockers and bupivacaine occur within the first ten minutes after injection. ${ }^{38}$

One of our study's limitations is the relatively small sample sizes used for animal experiments. While we chose a sample size of four to detect only large differences in nerve block duration and toxicity, we recognize that smaller - and perhaps clinically significant - differences may have been missed. It is also possible that larger sample sizes would reveal some functional predominance to the nerve blocks produced by our formulations. Another limitation is our somewhat restricted ability to determine the safety of our formulations with certainty; while we did not detect significant toxicity using methods that are validated in the literature - observation of behavior, contralateral blocks or death, histology and TEM - it is possible that studies using different methods may be beneficial. For example, other methods of nerve characterization, including quantification of neuropeptides such as substance $\mathrm{P}$ or calcitonin gene-related peptide, ${ }^{51,52}$ may show differences in nerve physiology after treatment with our formulations.

Our formulation could undergo modifications for enhanced performance in the clinical setting. We chose to use subclinical amounts of both drugs in part to facilitate the detection of their potential synergistic effect. However, the lack of toxicity detected in our animals indicates that these drug amounts may be further increased before appreciable toxicity occurs. In the case of TTX, which has a very limiting therapeutic 
index, future work may focus on combining an increase in drug dose with the use of a vehicle that releases the drug more slowly. In addition, an increased ratio of TTX to vehicle, without compromising release kinetics, may result in greater exposure of TTX to the nerve and further prolongation of nerve blockade.

This work suggests that capsaicin, and perhaps other compounds that act by a similar mechanism, may be used safely to prolong the duration of other encapsulated local anesthetics, particularly in formulations that include site-1 sodium channel blockers. Prolonged duration local anesthesia has been achieved by encapsulating site-1 sodium channel blockers and/or conventional local anesthetics. ${ }^{23,24,53}$ Those two classes of compounds show marked synergy in solution, ${ }^{19}$ and once encapsulated. ${ }^{24,54}$ Co-delivery with adjuvantic compounds, such as glucocorticoid receptor agonists, $\alpha 2$-adrenergic

agonists and other vasoconstrictors, free or encapsulated, singly or in combination, ${ }^{55}$ can dramatically prolong nerve blockade. It remains to be seen which of these formulations will be enhanced by addition of capsaicin. In that context, it is important to be mindful that there may be a ceiling to the effect of capsaicin beyond which its loading will incur local toxicity.

\section{Conclusion}

In conclusion, we have demonstrated that the combined delivery of capsaicin and TTX using a sustained-release system can achieve prolonged duration local anesthesia. Our formulation of combined drug-loaded liposomes has a number of properties that make it appealing for treating postoperative pain. In addition to prolonging the duration of analgesia otherwise produced by the administration of capsaicin only, TTX only, or other conventional local anesthetics, ${ }^{16,19,56}$ our formulation is easily injectable and results in no detectable local or systemic toxicity. Furthermore, our formulation may offer an alternative to opiate analgesics, which are associated with severe side effects ${ }^{57,58}$ and widespread misuse, abuse, addiction, and overdose. ${ }^{59}$ This work paves the way for the development of newer, safer, and more effective local anesthetics for postoperative pain management and other clinical applications. 


\section{References}

1. Møiniche S, Mikkelsen S, Wetterslev J, Dahl JB. A qualitative systematic review of incisional local anaesthesia for postoperative pain relief after abdominal operations. Br J Anaesth. 1998 Sep 19;81(3):377-83.

2. Zink W, Graf BM. Local anesthetic myotoxicity. Reg Anesth Pain Med. 2004 Mar 4;29(4):333-40.

3. Yang S, Abrahams MS, Hurn PD, Grafe MR, Kirsch JR. Local Anesthetic Schwann Cell Toxicity Is Time and Concentration Dependent. Reg Anesth Pain Med. 2011 Sep 20;36(5):444-51.

4. Albright GA. Cardiac arrest following regional anesthesia with etidocaine or bupivacaine. Anesthesiology. 1979 Oct 9;51(4):285-7.

5. Brown DL, Ransom DM, Hall JA, Leicht CH, Schroeder DR, Offord KP. Regional anesthesia and local anesthetic-induced systemic toxicity: seizure frequency and accompanying cardiovascular changes. Anesth Analg. 1995 Aug 22;81(2):321-8.

6. Binshtok AM, Bean BP, Woolf CJ. Inhibition of nociceptors by TRPV1-mediated entry of impermeant sodium channel blockers. Nature. 2007 Oct 4;449(7162):60710.

7. Hamalainen MM, Subieta A, Arpey C, Brennan TJ. Differential Effect of Capsaicin Treatment on Pain-Related Behaviors After Plantar Incision. J Pain. 2009 May 1;10(6):637-45.

8. Abdullah M, Mahowald M, Frizelle S, Dorman C, Funkenbusch S, Krug H. The effect of intra-articular vanilloid receptor agonists on pain behavior measures in a murine model of acute monoarthritis. J Pain Res. 2016 Aug 6; Volume 9:563-70.

9. Caterina MJ, Leffler A, Malmberg AB, Martin WJ, Trafton J, Petersen-Zeitz KR, et al. Impaired nociception and pain sensation in mice lacking the capsaicin receptor. Science. 2000 Apr 14;288(5464):306-13.

10. Fitzgerald M, Woolf CJ. The time course and specificity of the changes in the behavioural and dorsal horn cell responses to noxious stimuli following peripheral nerve capsaicin treatment in the rat. Neuroscience. 1982 May 12;7(9):2051-6.

11. Numazaki M, Tominaga T, Takeuchi K, Murayama N, Toyooka H, Tominaga M. 
Structural determinant of TRPV1 desensitization interacts with calmodulin. Proc Natl Acad Sci U S A. 2003 Jun 24;100(13):8002-6.

12. Szallasi A, Blumberg PM. Vanilloid (Capsaicin) receptors and mechanisms. Pharmacol Rev. 1999 Jun 12;51(2):159-212.

13. Jancśo G, Király E, Jancsó-Gábor A. Direct evidence for an axonal site of action of capsaicin. Naunyn Schmiedebergs Arch Pharmacol. 1980 Aug 12;313(1):91-4.

14. Gerner P, Binshtok AM, Wang C-F, Hevelone ND, Bean BP, Woolf CJ, et al. Capsaicin Combined with Local Anesthetics Preferentially Prolongs Sensory/Nociceptive Block in Rat Sciatic Nerve. Anesthesiology. 2008 Nov 11;109(5):872-8.

15. Colvin AC, Wang C-F, Soens MA, Mitani AA, Strichartz G, Gerner P. Prolonged Cutaneous Analgesia With Transdermal Application of Amitriptyline and Capsaicin. Reg Anesth Pain Med. 2011 May 11;36(3):236-40.

16. Kohane DS, Kuang Y, Lu NT, Langer R, Strichartz GR, Berde CB. Vanilloid receptor agonists potentiate the in vivo local anesthetic activity of percutaneously injected site 1 sodium channel blockers. Anesthesiology. 1999 Feb 11;90(2):52434.

17. Adams HJ, Blair MR, Takman BH. The Local Anesthetic Activity of Tetrodotoxin Alone and in Combination With Vasoconstrictors and Local Anesthetics. Anesth Analg. 1976 Jul 7;55(4):568-73.

18. Padera RF, Tse JY, Bellas E, Kohane DS. Tetrodotoxin for prolonged local anesthesia with minimal myotoxicity. Muscle Nerve. 2006 Dec 13;34(6):747-53.

19. Kohane DS, Yieh J, Lu NT, Langer R, Strichartz GR, Berde CB. A re-examination of tetrodotoxin for prolonged duration local anesthesia. Anesthesiology. $1998 \mathrm{Jul}$ 19;89(1):119-31.

20. Sakura S, Bollen AW, Ciriales R, Drasner K. Local anesthetic neurotoxicity does not result from blockade of voltage-gated sodium channels. Anesth Analg. 1995 Aug 11;81(2):338-46.

21. Lobo K, Donado C, Cornelissen L, Kim J, Ortiz R, Peake RWA, et al. A Phase 1, Dose-escalation, Double-blind, Block-randomized, Controlled Trial of Safety and Efficacy of Neosaxitoxin Alone and in Combination with $0.2 \%$ Bupivacaine, with 
and without Epinephrine, for Cutaneous Anesthesia. Anesthesiology. 2015 Oct 3;123(4):873-85.

22. Zimmer T. Effects of Tetrodotoxin on the Mammalian Cardiovascular System. Mar Drugs. 2010 Mar 19;8(3):741-62.

23. Rwei AY, Lee J-J, Zhan C, Liu Q, Ok MT, Shankarappa SA, et al. Repeatable and adjustable on-demand sciatic nerve block with phototriggerable liposomes. Proc Natl Acad Sci. 2015 Dec 7;112(51):15719-24.

24. Epstein-Barash H, Shichor I, Kwon AH, Hall S, Lawlor MW, Langer R, et al. Prolonged duration local anesthesia with minimal toxicity. Proc Natl Acad Sci U S A. 2009 Apr 28;106(17):7125-30.

25. Liu Q, Santamaria CM, Wei T, Zhao C, Ji T, Yang T, et al. Hollow Silica Nanoparticles Penetrate the Peripheral Nerve and Enhance the Nerve Blockade from Tetrodotoxin. Nano Lett. 2018 Jan 10;18(1):32-7.

26. Großkreutz J, Quasthoff S, Kühn M, Grafe P. Capsaicin blocks tetrodotoxinresistant sodium potentials and calcium potentials in unmyelinated $\mathrm{C}$ fibres of biopsied human sural nerve in vitro. Neurosci Lett. 1996 Jun 1;208(1):49-52.

27. Jancsó G, Lawson SN. Transganglionic degeneration of capsaicin-sensitive C-fiber primary afferent terminals. Neuroscience. 1990 May 8;39(2):501-11.

28. Jancsó G, Király E, Joó F, Such G, Nagy A. Selective degeneration by capsaicin of a subpopulation of primary sensory neurons in the adult rat. Neurosci Lett. 1985 Aug 30;59(2):209-14.

29. Yu S-Q, Premkumar LS. Ablation and Regeneration of Peripheral and Central TRPV1 Expressing Nerve Terminals and the Consequence of Nociception. Open Pain J. 2015 Mar 31;8(1):1-9.

30. Padera R, Bellas E, Tse JY, Hao D, Kohane DS. Local Myotoxicity from Sustained Release of Bupivacaine from Microparticles. Anesthesiology. 2008 May $1 ; 108(5): 921-8$.

31. Zimmermann M. Ethical guidelines for investigations of experimental pain in conscious animals. Pain. 1983 Jun 18;16(2):109-10.

32. Thalhammer JG, Vladimirova M, Bershadsky B, Strichartz GR. Neurologic evaluation of the rat during sciatic nerve block with lidocaine. Anesthesiology. 
1995 Apr 19;82(4):1013-25.

33. Bligh EG, Dyer WJ. A Rapid Method of Total Lipid Extraction and Purification. Can J Biochem Physiol. 1959 Aug 25;37(8):911-7.

34. McAlvin JB, Padera RF, Shankarappa SA, Reznor G, Kwon AH, Chiang HH, et al. Multivesicular liposomal bupivacaine at the sciatic nerve. Biomaterials. 2014 May 24;35(15):4557-64.

35. Hosmer DW, Lemeshow S, Sturdivant RX. Applied logistic regression. 3rd editio. New York: Wiley; 2013. 39-45 p.

36. Sokal RR, Rohlf FJ. Biometry: The Principles and practice of statistics in biological research. 3rd editio. W. H. Freeman; 1995. 229-41 p.

37. Conover WJ. Practical nonparametric statistics. 3rd editio. New York: Wiley; 1999. 288-96 p.

38. Kohane DS, Lu NT, Gökgöl-Kline AC, Shubina M, Kuang Y, Hall S, et al. The local anesthetic properties and toxicity of saxitonin homologues for rat sciatic nerve block in vivo. Reg Anesth Pain Med. 2000 Sep 25;25(1):52-9.

39. Santamaria CM, Zhan C, McAlvin JB, Zurakowski D, Kohane DS. Tetrodotoxin, Epinephrine, and Chemical Permeation Enhancer Combinations in Peripheral Nerve Blockade. Anesth Analg. 2017 Apr 8;124(6):1804-12.

40. McAlvin JB, Reznor G, Shankarappa SA, Stefanescu CF, Kohane DS. Local Toxicity from Local Anesthetic Polymeric Microparticles. Anesth Analg. 2013 Apr 8;116(4):794-803.

41. Feirabend HKP, Choufoer H, Ploeger S. Preservation and Staining of Myelinated Nerve Fibers. Methods. 1998 Jun 8;15(2):123-31.

42. Kissin I, Freitas CF, Mulhern HL, DeGirolami U. Sciatic Nerve Block with Resiniferatoxin: An Electron Microscopic Study of Unmyelinated Fibers in the Rat. Anesth Analg. 2007 Sep 8;105(3):825-31.

43. Spencer PS, Lieberman AR. Scanning electron microscopy of isolated peripheral nerve fibres. Zeitschrift fur Zellforsch und Mikroskopische Anat. 1971 Oct 24;119(4):534-51.

44. Schmalbruch H. Fiber composition of the rat sciatic nerve. Anat Rec. 1986 May $26 ; 215(1): 71-81$. 
45. Czaja K, Burns GA, Ritter RC. Capsaicin-induced neuronal death and proliferation of the primary sensory neurons located in the nodose ganglia of adult rats. Neuroscience. 2008 Jun 23;154(2):621-30.

46. Chard PS, Bleakman D, Savidge JR, Miller RJ. Capsaicin-induced neurotoxicity in cultured dorsal root ganglion neurons: involvement of calcium-activated proteases. Neuroscience. 1995 Apr 30;65(4):1099-108.

47. Simone DA, Nolano M, Johnson T, Wendelschafer-Crabb G, Kennedy WR. Intradermal injection of capsaicin in humans produces degeneration and subsequent reinnervation of epidermal nerve fibers: correlation with sensory function. J Neurosci. 1998 Nov 1;18(21):8947-59.

48. Neubert JK, Karai L, Jun JH, Kim H-S, Olah Z, Iadarola MJ. Peripherally induced resiniferatoxin analgesia. Pain. 2003 Jul 7;104(1-2):219-28.

49. Yoshimura N. Bladder afferent pathway and spinal cord injury: possible mechanisms inducing hyperreflexia of the urinary bladder. Prog Neurobiol. 1999 Jun 1;57(6):583-606.

50. Kwong K, Lee L-Y. Prostaglandin E2 potentiates a TTX-resistant sodium current in rat capsaicin-sensitive vagal pulmonary sensory neurones. J Physiol. 2005 Apr 15;564(Pt 2):437-50.

51. Ainsworth A, Hall P, Wall PD, Allt G, MacKenzie ML, Gibson S, et al. Effects of capsaicin applied locally to adult peripheral nerve. II. Anatomy and enzyme and peptide chemistry of peripheral nerve and spinal cord. Pain. 1981 Dec 14;11(3):379-88.

52. Carr PA, Yamamoto T, Nagy JI. Calcitonin gene-related peptide in primary afferent neurons of rat: co-existence with fluoride-resistant acid phosphatase and depletion by neonatal capsaicin. Neuroscience. 1990 Jun 7;36(3):751-60.

53. Ilfeld BM, Malhotra N, Furnish TJ, Donohue MC, Madison SJ. Liposomal Bupivacaine as a Single-Injection Peripheral Nerve Block. Anesth Analg. 2013 Nov 28;117(5):1248-56.

54. Kohane DS, Smith SE, Louis DN, Colombo G, Ghoroghchian P, Hunfeld NGM, et al. Prolonged duration local anesthesia from tetrodotoxin-enhanced local anesthetic microspheres. Pain. 2003 Jul 18;104(1-2):415-21. 
55. Rwei AY, Sherburne RT, Zurakowski D, Wang B, Kohane DS. Prolonged Duration Local Anesthesia Using Liposomal Bupivacaine Combined With Liposomal Dexamethasone and Dexmedetomidine. Anesth Analg. 2018 Apr 7;126(4):1170-5.

56. Becker DE, Reed KL. Essentials of local anesthetic pharmacology. Anesth Prog. 2006 Jun 7;53(3):98-108-10.

57. Dahan A, Aarts L, Smith TW. Incidence, Reversal, and Prevention of Opioidinduced Respiratory Depression. Anesthesiology. 2010 Jan 3;112(1):226-38.

58. Chidambaran V, Olbrecht V, Hossain M, Sadhasivam S, Rose J, Meyer MJ. Risk Predictors of Opioid-Induced Critical Respiratory Events in Children: Naloxone Use as a Quality Measure of Opioid Safety. Pain Med. 2014 Dec 1;15(12):213949.

59. Kharasch ED, Brunt LM. Perioperative Opioids and Public Health. Anesthesiology. 2016 Apr 1;124(4):960-5. 


\title{
Glossary of Abbreviations
}

\author{
ANOVA: analysis of variance \\ CAP: capsaicin \\ DLS: dynamic light scattering \\ DLPC: 1,2-dilinoleoyl-sn-glycero-3-phosphocholine \\ DSPC: 1,2-distearoyl-sn-glycero-3-phosphocholine \\ DSPG: 1,2-distearoyl-sn-glycero-3-phospho-(1'-rac-glycerol) \\ H\&E: haematoxylin and eosin \\ Lipo: liposome \\ PBS: phosphate-buffered saline \\ SD: standard deviation \\ TEM: transmission electron microscopy \\ TTX: tetrodotoxin
}

